\title{
ON PERMUTATIONS AND PERMUTATION POLYTOPES
}

\author{
H.P. Young*
}

RR-78-5

March 1978

This work was supported in part by the National Science Foundation under Grant MPS 75-07414 with the Graduate School of the City University of New York.

*Graduate School and University Center, City University of New York, and IIASA.

Research Reports provide the formal record of research conducted by the International Institute for Applied Systems Analysis. They are carefully reviewed before publication and represent, in the Institute's best judgment, competent scientific work. Views or opinions expressed therein, however, do not necessarily reflect those of the National Member Organizations supporting the Institute or of the Institute itself.

International Institute for Applied Systems Analysis A-2361 Laxenburg, Austria 
Jeanne Anderer, editor

Martin Schobel, graphics

Printed by NOVOGRAPHIC

Maurer-Lange-Gasse 64

1238 Vienna

Copyright (C) 1978 IIASA

All rights reserved. No part of this publication may be reproduced or transmitted in any form or by any means, electronic or mechanical, including photocopy, recording, or any information storage or retrieval system, without permission in writing from the publisher. 


\section{PREFACE}

The development of better methods to compute optimal solutions to models is a basic task in the System and Decision Sciences Area. Of particular interest are linear models with linear constraints. An important problem here is to be able to characterize when one solution is "adjacent" to another, since most practical algorithms for linear models operate by moving from one solution to an adjacent "better" one.

In this paper a convenient criterion is formulated to characterize adjacency for constraint systems that arise from orderings. The results have application to models in which the object is to find a best ordering of alternatives using some linear criterion, for example. In particular, it was used as a technique for studying collective decision criteria (H.P. Young and A. Levenglick, $A$ Consistent Extension of Condorcet's Election Principle, RR-77-15, International Institute for Applied Systems Analysis, Laxenburg, Austria). 


\section{SUMMARY}

Permutation polytopes arise in a class of problems in which the objective is to find an optimal complete ordering of some given alternatives, subject to a linear objective criterion. In this paper an easy characterization is given of neighbors on permutation polytopes. Using this characterization it is shown that the graph of any such polytope is Hamiltonian, and that the diameter is two.

The methods used are combinatorial in nature. 



\section{On Permutations and Permutation Polytopes}

\section{INTRODUCTION}

A permutation, or linear ordering, on the $\mathrm{n}$ symbols $1,2, \ldots, \mathrm{n}$ will be denoted by the $n$-tuple $\sigma=(\sigma(1), \sigma(2), \ldots, \sigma(n))$. We also write $h \sigma k$ if $h$ precedes $k$ in the order $\sigma$. One useful algebraic representation of a permutation is by a permutation matrix. Here we shall consider another way of representing permutations by $(0,1)$ matrices that has application to a variety of problems including computer design [2] and collective decision making [5].

For any linear ordering $\sigma=(\sigma(1), \sigma(2), \ldots, \sigma(n))$ let $\mathrm{E}^{\sigma}$ be the $\mathrm{n} \times \mathrm{n}$ matrix with 1 in position $(i, j)$ if $i \sigma j$ and 0 otherwise. The permutation polytope $\mathrm{P}_{\mathrm{n}}$ is defined to be the set of all convex combinations of the matrices $E^{\sigma}, \sigma$ a permutation of order $n$. It is an unsolved problem to determine the complete set of inequalities defining a general $\mathrm{P}_{\mathrm{n}}$ (those proposed in [1] being insufficient). On the other hand various characterizations of neighbors on these polytopes are known. Here we shall give a computationally easy way of recognizing neighbors in terms of the structure of the permutations. This approach also reveals some facts about the structure of a class of graphs (implicitly introduced in [3,4] called transposition graphs that are intimately connected with adjacency questions on the graph of $\mathrm{P}_{\mathrm{n}}$.

\section{BLOCKS}

An interval of a permutation $\sigma=(\sigma(1), \sigma(2), \ldots, \sigma(n))$ is a subsequence of form $(\sigma(i), \sigma(i+1), \ldots, \sigma(j))$. We also allow the empty subsequence. Let $e=(1,2, \ldots, n)$ be the identity permutation. A block of $\sigma$ is any interval of $\sigma$ that can be rearranged to be an interval of e. A common interval of $\sigma$ and $e$ is an interval (without rearrangement) of both $\sigma$ and $e$.

Example 1. Let $e=(1,2,3, \ldots, 10,11)$ and let $\sigma=(2,10,4,11$, $6,5,8,7,9,3,1)$. The blocks of $\sigma$, aside from $\phi$ and the singletons, are: 


$$
\begin{aligned}
& \beta_{1}=(2,10,4,11,6,5,8,7,9,3) \\
& B_{2}=\sigma \\
& \beta_{3}=(10,4,11,6,5,8,7,9) \\
& \beta_{4}=(10,4,11,6,5,8,7,9,3) \\
& \beta_{5}=(6,5) \\
& \beta_{6}=(6,5,8,7) \\
& \beta_{7}=(6,5,8,7,9) \\
& B_{8}=(8,7) \\
& \beta_{9}=(8,7,9)
\end{aligned}
$$

The transposition set of $\sigma, \mathbf{S}(\sigma)$, is the set of all unordered pairs $\{i, j\} i \neq j$ inverted by $\sigma: s(\sigma)=\{\{i, j\}: i<j$ and $j \sigma i\}$. The transposition set of a block $\beta$ of $\sigma, s(\beta)=\{\{i, j\}: i, j \varepsilon \beta$, $\mathbf{i}<\mathbf{j}$ and $j \sigma i\}$.

Notice that for any two blocks $\beta_{1}, \beta_{2}$ of $\sigma, \beta_{1} \cap \beta_{2}$ is also a block and

$$
S\left(\beta_{1} \cap \beta_{2}\right)=S\left(\beta_{1}\right) \cap S\left(\beta_{2}\right)
$$

Let $L_{\sigma}=\left\{S: S=S\left(\beta_{i}\right)\right.$ for some block $\beta_{i}$ of $\left.\sigma\right\}$. Then the elements of $\mathrm{L}_{\sigma}$, ordered by inclusion, form a lattice, called a permutation lattice. In general, l.u.b. $\left\{\mathbf{s}_{\mathbf{i}}\right\}_{\mathrm{s}_{i}} \in L_{\sigma}^{2} \cup_{\mathbf{i}} \mathrm{s}_{\mathbf{i}}$, but equality
may not hold.

For the $\sigma$ of Example 1, we obtain the following lattice.

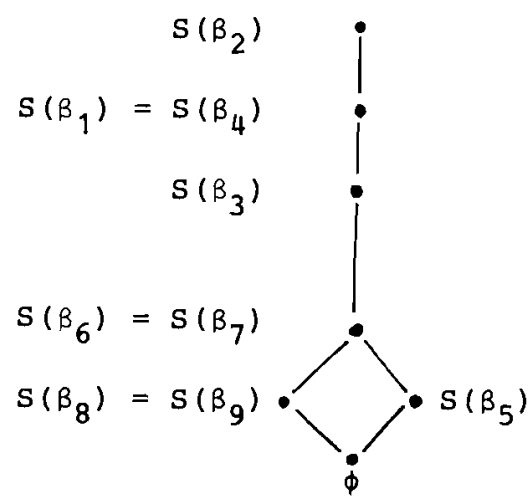




\section{CHARACTERIZATION OF NEIGHBORS BY BLOCKS}

Two permutations $\sigma$ and $\tau$ are said to be neighbors written $\sigma \sim \tau$ if $E^{\sigma}$ and $E^{\top}$ are neighboring extreme points on $\mathrm{P}_{\mathrm{n}}$. We may now give the following easy characterization of neighbors on permutation polytopes.

$$
\begin{aligned}
& \sigma \neq e \text { is a neighbor of } e \text { on } P_{n} \text { if and only if } \\
& L_{\sigma} \text { has a unique nonempty element. }
\end{aligned}
$$

This will be established below as a consequence of Theorem 1 .

For the moment let us notice that this criterion leads to a highly efficient method for checking whether a given $\sigma$ is a neighbor of e.

First, find the least $p^{0}, 1 \leq p^{0} \leq n$ such that $\sigma\left(p^{0}\right) \neq p^{0}$ (if none exists, $\sigma=e)$. Next, order the pairs $(p, q), p^{0} \leq p<q \leq n$, lexicographically. For each successive pair we check to see whether the interval $(\sigma(p), \ldots, \sigma(q))$ is a block: this is true iff $q-p=M-m$, where $M=\max _{p<i<q} \sigma(i), m=\min _{p<i<q} \sigma(i)$. Moreover, this block is nontrivial (that is, it is not an interval of e) if $M \neq \sigma(q)$. If $\left(p^{\prime}, q^{\prime}\right)$ is the first pair for which a nontrivial block is found, we check that $\sigma(i)=i$ for $q^{\prime}<i<n$ : if this is false, $\sigma$ is not a neighbor of $e$. If true, repeat the above process for successive pairs $(p, q),\left(p^{\prime}+1\right) \leq p<q \leq q^{\prime}$ : if any nontrivial block is found, $\sigma$ is not a neighbor of e; otherwise $\sigma$ is a neighbor of $e$.

Notice that the computation of the new $M$ and $n$ at each stage is easy, since the pairs $(p, q)$ are taken in lexicographic order. The number of pairs $(p, q)$ that have to be considered is at most $\left(\begin{array}{l}n \\ 2\end{array}\right)$.

\section{CHARACTERIZATION OF NEIGHBORS BY GRAPHS}

A second way of characterizing neighbors on permutation polytopes is based on a certain class of comparability graphs. An undirected graph $G$ is said to be a comparability graph if its edges can be directed so as to form a strict partial order $\theta$ : i.e., 
(i) exactly one of $(i, j),(j, i) \varepsilon \theta$ iff $\{i, j\}$ is an edge of $G$, (ii) $(i, j),(j, k) \varepsilon \theta$ implies $(i, k) \varepsilon \theta$.

Given a permutation $\sigma=(\sigma(1), \ldots, \sigma(n))$, the permutation graph [3] of $\sigma, G_{\sigma}$, is defined to be the undirected graph with vertex set $\{1,2, \ldots, n\}$ and edge set $S(\sigma)$. Notice that $G_{\sigma}$ is a comparability graph by the partial order $\theta=\{(j, i): i<j$ and $\{i, j\} \in S(\sigma)\}$ (or the reverse order $\bar{\theta}$ ). Similarly, $G_{\sigma}^{C}$ is a comparability graph for the order $\theta^{\prime}=\{(i, j): i<j$ and $[i, j] \not t(\sigma)\}$. Conversely, if $G$ is some graph such that both $G$ and $G^{C}$ are comparability graphs then $G=G_{\sigma}$ for some $\sigma[3]$.

Given permutation $\sigma$, a graph related to $G_{\sigma}$ is the transposition set $F_{\sigma}$ defined as follows: $\Gamma_{\sigma}$ has vertex set $S(\sigma)$, and $\{i, j\} \varepsilon S(\sigma)$ is adjacent to $\{j, k\} \varepsilon S(\sigma)$ (written $\{i, j\} \Gamma\{j, k\}$ ) if and only if $i \neq k$ and $\{i, k\} \&(\sigma)$; these are all the adjacencies in $\Gamma_{\sigma}$.

The following connection between $\Gamma_{\sigma}$ and neighbors on the permutation polytope is due to Alan Hoffman.

$$
\sigma \neq e \text { is a neighbor of } e \text { if and only if } \Gamma_{\sigma} \text { is connected }
$$

To prove sufficiency, suppose $\Gamma_{\sigma}$ is connected and $\sigma \neq \mathrm{e}$ is not a neighbor of e. Then $S(\sigma)=\bigcup_{r=1}^{S} S\left(\pi_{r}\right)$ for distinct permutations $\pi_{r} \neq \sigma, r \geq 2$.

Since $\Gamma_{\sigma}$ is connected, there is $\pi_{r} \neq \pi_{r^{\prime}}$ and $\{i, j\} \varepsilon s\left(\pi_{r}\right)-$ $S\left(\pi_{r^{\prime}}\right),\{j, k\} \in S\left(\pi_{r^{\prime}}\right)$, such that $\{i, j\} \Gamma\{j, k\}, i . e .\{i, k\} \not t S(\sigma)$. Hence $\{i, k\} \not \subset S\left(\pi_{r}\right) \cup s\left(\pi_{r}\right)$. Hence, of $i, j, k, \pi_{r}$ ' reverses only $j$ and $k$, and $\pi_{r}$ reverses $j$ and $i$ but not $i$ and $k$. Therefore, either $i<j<k$ or $k<j<i$. But $\sigma$ reverses $\{i, j\}$ and $\{j, k\}$ and not $\{i, k\}$, an absurdity. The converse will be established as a consequence of the following connection between $\Gamma_{\sigma}$ and the permutation lattice $\mathrm{L}_{\sigma}$.

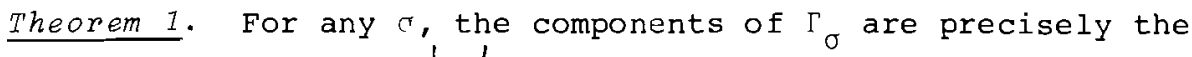
nonempty sets of form $s-\underbrace{}_{S_{i}} S_{i} s_{i}$ where $s \varepsilon L_{\sigma}$.

$$
s_{i} \in L_{\sigma}
$$

We proceed by several lemmas. 
Lemma 1.1. If $C$ is any component of $\Gamma_{\sigma}$ and $C \cap S \neq \phi$ for some $S \in L_{\sigma}$, then $C \subseteq S$.

Proof: If $C \notin S$ then $\{i, j\} \Gamma\{j, k\}$ for some $\{i, j\} \varepsilon C \cap S$ and some $\{j, k\} \in C-S$. Let $S=S(\beta), \beta=(\sigma(p), \ldots, \sigma(q))$; then $i, j \varepsilon \beta$, $k \notin \beta$. Since $\sigma$ reverses $j$ and $k$ it must also reverse $i$ and $k$, whence $\{i, k\} \varepsilon S$, a contradiction.

Recall that $G_{\sigma}$ has vertex set $V=\{1,2, \ldots, n\}$ and edge set $S(\sigma)$.

Following [3], we say that a subset $V^{\prime} \subseteq \mathrm{V}$ is uniformly hinged (u.h.) in $G_{\sigma}$ if for every $k \notin V^{\prime}$, either $\{k, i\} \varepsilon S(\sigma)$ for $a l z$ i $\varepsilon V^{\prime}$ or $\{k, i\} \varepsilon S(\sigma)$ for no $i \varepsilon V^{\prime}$. Clearly any block of $\sigma$ regarded as a set of vertices of $G_{\sigma}$ is $u . h .$, but not every $u . h$. set constitutes a block. For any $V^{\prime} \subseteq V$ and any permutation $\pi$ define $c l_{\pi}\left(V^{\prime}\right)$ as the smallest interval of $\pi$ containing $V^{\prime}$.

Lemma 1.2. If $V^{\prime}$ is $\mathrm{u} . \mathrm{h}$. in $\mathrm{G}_{\sigma}$ then, as sets, $\mathrm{cl}_{\mathrm{e}}\left(\mathrm{V}^{\prime}\right)$ equals $c l_{\sigma}\left(V^{\prime}\right)$, in which case $c l_{\sigma}\left(V^{\prime}\right)$ is a block of $\sigma$.

Proof: Suppose $V^{\prime}$ is u.h., and let $c \ell_{\sigma}\left(V^{\prime}\right)=(\sigma(p), \ldots, \sigma(q))$. Then $\sigma(p), \sigma(q) \in V^{\prime}$ by definition. If i $\varepsilon c \ell_{\sigma}\left(V^{\prime}\right)-V^{\prime}$, and $\sigma(p)>\sigma(q)$, then $u . h$. implies $\sigma(p)>i>\sigma(q)$; similarly, if $\sigma(p)<$ $\sigma(q)$ then $\sigma(p)<i<\sigma(q)$. Hence $c l_{\sigma}\left(V^{\prime}\right) \subseteq c l_{e}\left(V^{\prime}\right)$. On the other hand, if $i \varepsilon c h\left(V^{\prime}\right)-V^{\prime}$ then for some $h, k \varepsilon V^{\prime}$ we have $h<i<k$. Thus $i$ cannot precede (or follow) both $h$ and $k$ in $\sigma$ without contradicting u.h., hence $i \varepsilon c l_{\sigma}\left(V^{\prime}\right)$ and $c l_{\sigma}\left(V^{\prime}\right)=c l_{e}\left(V^{\prime}\right)$.

For any subset of edges $E \cong S(\sigma)$, let $V(E) \subseteq V$ be the set of endpoints of edges in $\mathrm{E}$. The next two results are immediate consequences of Lemmas 2 and 4, respectively in [3].

Lemma 1.3. If $\mathrm{C}$ is a component of $\Gamma_{\sigma}$ then $\mathrm{V}(\mathrm{C})$ is $\mathrm{u} . \mathrm{h}$. in $\mathrm{G}_{\sigma}$.

Lemma 1.4 . If $C$ and $C^{\prime}$ are distinct components of $\Gamma_{\sigma}$ then $\mathrm{V}(\mathrm{C}) \neq \mathrm{V}\left(\mathrm{C}^{\prime}\right)$.

Proof of Theorem 1: By Lemma 1.1, any component that meets

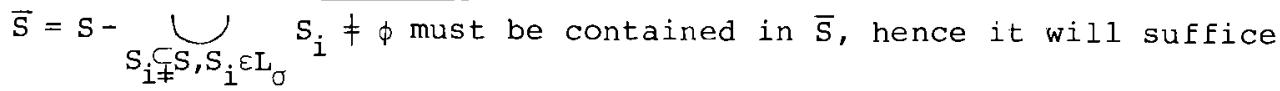
to show that $\bar{S}$ is connected in $\Gamma_{\sigma}$. 
Let $\mathrm{C}$ be any component of $\Gamma_{\sigma}$ contained in $\overline{\mathrm{s}}$, and let $\mathrm{W}=\mathrm{V}(\mathrm{C})$. Since $w$ is u.h., Lemma 1.2 implies $c \ell_{\sigma}(W)=\beta$ is a block of $\sigma$, say $\beta=(\sigma(p), \ldots, \sigma(q))$.

Suppose $\beta-W \neq \phi$. (Here we think of $\beta$ as a set as well as a sequence.)

Let $h \in \beta-W$ and

$$
\begin{aligned}
& \mathrm{W}_{\mathrm{h}}^{+}=\{\mathrm{k} \in \mathrm{W}: \mathrm{k} \sigma \mathrm{h}\}, \\
& \mathrm{W}_{\mathrm{h}}^{-}=\{\mathrm{k} \in \mathrm{W}: \mathrm{h} \sigma \mathrm{k}\} .
\end{aligned}
$$

Notice that since $\sigma(p), \sigma(q) \varepsilon w, w_{h}^{+}, w_{h}^{-} \neq \phi ;$ moreover $w_{h}^{+}$and $w_{h}^{-}$ are each u.h. Therefore $\beta_{h}^{+}=c l_{\sigma}\left(W_{h}^{+}\right)$and $\beta_{h}^{-}=c l_{\sigma}\left(W_{h}^{-}\right)$are nonempty disjoint blocks.

In general, for any disjoint $V^{\prime}, V^{\prime \prime} \subseteq V^{\prime}$, let $\left(V^{\prime}, V^{\prime \prime}\right)$ denote the set of edges of $G_{\sigma}$ with one end in $V^{\prime}$ and the other end in $V^{\prime \prime}$.

By Lemma 1.1, $C \cap S\left(\beta_{h}^{+}\right)=\phi$ and $C \cap S\left(\beta_{h}^{-}\right)=\phi$, hence $C \subseteq\left(W_{h}^{+}, W_{h}^{-}\right)$.

Suppose now that some $k \varepsilon \beta_{h}^{+}-W_{h}^{+} \neq \phi$. Then by a similar argument we have $c \subset\left(w_{k^{+}}^{+}, w_{k}^{-}\right)$. By choice of $k, w_{h}^{+}-w_{h}^{+} \neq \phi$, say $\ell \in W_{h}^{+}-W_{k}^{+}$. Then $\ell \varepsilon W_{k}^{-}$and $\ell$ are the endpoints of some edge in $c$. The other endpoint, $\ell^{\prime}$, is therefore in $\mathrm{w}_{\mathrm{k}}^{+} \varsigma \mathrm{w}_{\mathrm{h}}^{+}$, but then both ends are in $W_{h}^{+}$, a contradiction. Therefore $B_{h}^{+}=W_{h}^{+}$is a block and similarly $\beta_{h}^{-}=w_{h}^{-}$is a block; say $\beta_{h}^{+}=\left(\sigma(p), \ldots, \sigma\left(p^{\prime}\right)\right)$, $\beta_{h}^{-}=\left(\sigma\left(q^{\prime}\right), \ldots, \sigma(q)\right)$, and let $\alpha=\left(\sigma\left(p^{\prime}+1\right), \ldots, \sigma\left(q^{\prime}-1\right)\right) \neq \phi$. Since $C \neq \phi$ and $C \subseteq\left(\beta_{h}^{+}, \beta_{h}^{-}\right)$we must have $\beta_{h}^{+}>\beta_{h}^{-}$(i.e., i>j for al1 i $\left.\varepsilon \beta_{h}^{+}, j \varepsilon \beta_{h}^{-}\right)$. Further, since $w=\beta_{h}^{+} \cup \beta_{h}^{-}$is u.h. we have $\mathrm{P}_{\mathrm{h}}^{+}>\alpha>\beta_{\mathrm{h}^{-}}$, so $\alpha$ is also a nonempty block of $\sigma$.

In summary, for any component $c \subseteq \bar{s}$, if $V(C)=W$ and $\beta=c_{\sigma}(W)$, then either $W=\beta$ is a block or $\beta$ has form $\beta=\left(W^{+}, \alpha, W^{-}\right)$where $\mathrm{W}^{+}>\alpha>\mathrm{W}^{-}$are each nonempty blocks.

If $\overline{\mathrm{S}}$ is not connected then there is another component $\tilde{\mathrm{C}}$ of $\Gamma_{\sigma}$ such that $C \neq \tilde{C} \subseteq \bar{S}$ and corresponding $\tilde{W}, \tilde{\beta}$. By definition of $\bar{S}, S(\tilde{B})=S(\beta)=\bar{S}$, so $\beta=\tilde{\beta}$. 
Now $W=\beta=\tilde{\beta}=\tilde{W}$ is impossible by Lemma 1.4. Hence say, without loss of generality, that either

$$
\beta=\left(W^{+}, \alpha, W^{-}\right)=\widetilde{W},
$$

or

$$
\beta=\left(\mathrm{W}^{+}, \alpha, \mathrm{W}^{-}\right)=\left(\tilde{\mathrm{W}}^{+}, \tilde{\alpha}, \tilde{\mathrm{W}}^{-}\right)=\tilde{\beta},
$$

where $\mathrm{W}^{+}>\alpha>\mathrm{W}^{-}$and $\tilde{\mathrm{W}}^{+}>\tilde{\alpha}>\tilde{\mathrm{W}}^{-}$and each is a nonempty block.

In the first case notice that both $\left(w^{+}, \alpha\right)$ and $\left(\alpha, w^{-}\right)$are blocks. Choose any $k \varepsilon \alpha$; then $\{j, k\} \varepsilon \tilde{c}$ for some $j$ and either $\{j, k\} \varepsilon S\left(W^{+}, \alpha\right)$ or $\{j, k\} \in S\left(\alpha, W^{-}\right)$. But neither $S\left(W^{+}, \alpha\right)$ nor $S\left(\alpha, w^{-}\right)$ equals $s$, contradicting the definition of $\bar{s}$.

In the second case suppose $\mathrm{W}^{+} \neq \tilde{W}^{+}$; without loss of generality $\mathrm{w}^{+} \subsetneq \mathrm{W}^{+}$. Then $\left(\tilde{\mathrm{W}}^{+}-\mathrm{W}^{+}, \alpha, \tilde{W}^{-}\right)=\delta$ is a block. For any $k \varepsilon \tilde{W}^{+}-W^{+},\{k, j\} \varepsilon \tilde{C}$ for some $j$ and either $\{k, j\} \varepsilon s\left(W^{+}\right)$or $\{k, j\}$ $\varepsilon S(\delta)$. Since neither $S(\delta)$ nor $S\left(W^{+}\right)$equals $S$, this contradicts the definition of $\overline{\mathrm{S}}$. Thus $\mathrm{W}^{+}=\tilde{\mathrm{W}}^{+}$, and likewise $\mathrm{W}^{-}=\tilde{\mathrm{W}}^{-}$. But then $W=\tilde{W}$, contradicting Lemma 1.4 .0

The proof of statement (1) is now as follows.

If $L_{\sigma}$ has one nonempty element $s$, then $S=\bar{s}$ is the unique component of $\Gamma_{\sigma}$; hence by the argument following (2) $\sigma$ is a neighbor of e. If on the other hand $L_{\sigma}$ has distinct, nonempty elements, let $s_{0}=S(\beta)$ be a minimal nonempty element of $L_{\sigma}$. Then within $\beta$ we may rearrange the elements to be an interval of $e$, and the resulting permutation $\sigma^{\prime}$ satisfies $s\left(\sigma^{\prime}\right)=S(\sigma)-s_{0}$. Likewise we may rearrange $\sigma$ to agree with e everywhere except within $\beta$, and the resulting $\sigma^{\prime \prime}$ has $s\left(\sigma^{\prime \prime}\right)=s_{0}$. But then $E^{\sigma^{\prime}}+$ $E^{\sigma "}=E^{\sigma}+E^{e}$, showing that $\sigma$ is not a neighbor of e. Statement (2) now follows at once also, since if $\sigma$ is a neighbor of $e$, then $L_{\sigma}$ has a unique nonempty element; hence by Theorem $1 \Gamma_{\sigma}$ is connected.

Example 2. For $\mathrm{n}=3$, the neighbors of $\mathrm{e}=(1,2,3)$ are seen by inspection to be: $(2,1,3),(2,3,1),(1,3,2),(3,1,2)$. 
For $\mathrm{n}=4$, the neighbors of $\mathrm{e}=(1,2,3,4)$ are seen by inspection to be the following twelve permutations:

$\begin{array}{llll}(1,2,4,3) & (2,1,3,4) & (3,1,2,4) & (4,1,2,3) \\ (1,3,2,4) & (2,3,1,4) & (3,1,4,2) \\ (1,3,4,2) & (2,3,4,1) & (3,4,1,2) \\ (1,4,2,3) & (2,4,1,3) & \end{array}$

\section{THE CIRCUMFERENCE OF ${ }^{\mathrm{p}} \mathrm{n}$}

The graph of $\mathrm{P}_{\mathrm{n}}, \mathrm{H}\left(\mathrm{P}_{\mathrm{n}}\right)$, is the undirected graph whose vertices are the permutations $\sigma=(\sigma(1), \ldots, \sigma(n))$, where $\sigma$ and $\tau$ are adjacent if and only if $\sigma \neq \tau$ and $\sigma$ is a neighbor of $\tau$.

Theorem 22. The graph $\mathrm{H}\left(\mathbf{P}_{\mathbf{n}}\right)$ is Hamiltonian for any $\mathrm{n} \geq 3$.

Proof: For $\mathrm{n}=3$ a Hamiltonian circuit is

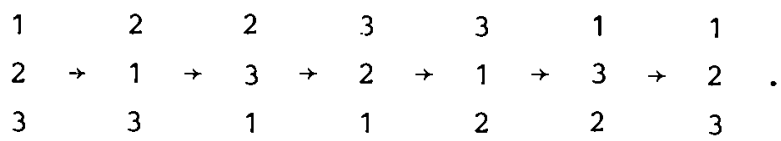

Suppose the result has been proved for $\mathrm{n}-1$, and let $\tau, \ldots$, $\tau_{(n-1)}{ }^{\tau}{ }_{1}$ be a Hamiltonian circuit on the permutations $\tau_{i}$ of $1,2, \ldots, n-1$. For each odd $i, 1 \leq i \leq(n-1) !$, let $\tau_{i}=\left(\tau_{i}(1), \ldots\right.$, $\tau_{i}(n-1)$, and for each $k, 1 \leq k \leq n$ define the permutations $\sigma_{i k}$ of $1,2, \ldots, \mathrm{n}$ as follows:

$$
\begin{aligned}
& \sigma_{i 1}=\left(\tau_{i}(1), \ldots, \tau_{i}(n-1), n\right) \\
& \sigma_{i 2}=\left(\tau_{i}(1), \ldots, n, \tau_{i}(n-1)\right) \\
& \vdots \\
& \sigma_{i n}=\left(n, \tau_{i}(1), \ldots, \tau_{i}(n-1)\right) .
\end{aligned}
$$

For $\mathrm{i}$ even, let 


$$
\begin{aligned}
& \sigma_{i 1}=\left(n, \tau_{i}(1), \ldots, \tau_{i}(n-1)\right) \\
& \sigma_{i 2}=\left(\tau_{i}(1), n, \ldots, \tau_{i}(n-1)\right) \\
& \vdots \\
& \sigma_{i n}=\left(\tau_{i}(1), \ldots, \tau_{i}(n-1), n\right) .
\end{aligned}
$$

Since $(n-1)$ ! is even, (1) implies that $\sigma_{11}, \sigma_{12}, \ldots, \sigma_{1 n}, \sigma_{21}, \ldots, \sigma_{2 n}$, $\sigma(n-1) ! n^{\prime} \sigma_{11}$ is a Hamiltonian circuit in $H\left(P_{n}\right) \cdot \sigma$

It will be noticed that each successive pair of permutations on the Hamiltonian circuit are "close" neighbors in the sense that they differ by just one adjacent transposition. This construction is known in combinatorics, see for example $[6,7]$.

\section{AN INDUCTIVE LEMMA}

Pick $m, 1 \leq m \leq n$, and let $m_{1}=m-1, m_{2}=m+1 . \quad$ (If $m=1$, only $\mathrm{m}_{2}$ is defined, and if $\mathrm{m}=\mathrm{n}$ only $\mathrm{m}_{1}$ is defined.)

Given any permutation $\pi$, let $\hat{\pi}$ be the permutation on $n-1$ symbols obtained from $\pi$ by removing $m$ from the sequence. Thus, if $\pi=(2,3,5,1,4)$ and $m=3$ then $\hat{e}=(1,2,4,5)$ and $\hat{\pi}=(2,5,1,4)$.

The $\mathrm{n}-1$ elements of $\hat{\pi}$ we call dots and the $\mathrm{n}$ positions defined between the dots or at either end of the dots we call slots. Given any dot sequence $\beta$ (i.e., an interval of $\hat{\pi}$, ) where $|\beta|=k$, let $(\beta)$ denote the sequence of $k$ dots and $k-1$ slots beginning with the first dot in $\beta$ and ending with the last dot in $\beta$; let $(\beta)$ denote the sequence with $k$ dots and $k$ slots consisting of ( $\beta$ ) and the end slot to the right of $\beta$; let $[\beta)$ be $(\beta)$ and the end slot to the left of $\beta$, and let $[\beta]$ be $(\beta)$ together with both end slots. We call these open, half-open and closed slot-dot sequences, respectively. We adopt the convention that if $\beta$ is the empty sequence then $[\beta]$ consists of one slut, and the others are empty. We also write $m \varepsilon(\beta), m \varepsilon[\beta]$ etc., when we mean that $\mathrm{m}$ occupies a slot in that sequence. 
Let $\gamma_{1}$ be the longest initial interval of $\hat{\pi}$ that is also an initial interval of $\hat{e}, \gamma_{2}$ the longest terminal interval of $\hat{\pi}$ that is also a terminal interval of $\hat{e}$ (either may be empty). $\hat{\pi}=\gamma_{1} \hat{\hat{\pi}} \gamma_{2}$ where $\hat{\pi}$ is a block of $\hat{\pi}$ (relative to $\hat{e}$ ) and $S(\hat{\pi})=S(\hat{\pi})$. Define $\delta_{1}$ to be the longest common interval of $\hat{\pi}$ and $\hat{e}$ ending with $\mathrm{m}_{1}, \delta_{2}$ to be the longest common interval of $\hat{\pi}$ and $\hat{\mathrm{e}}$ beginning with $\mathrm{m}_{2}$. (If $\mathrm{m}=1$ or $\mathrm{m}=\mathrm{n}$, one of these sequences is vacuous.)

Lemma 3.1. Let $\hat{\pi}$ be a neighbor of $\hat{e}$. Then $\pi$ is a neighbor of $e$ if and only if

(i) $\mathrm{m} \not\left[\delta_{1}\right), \mathrm{m} \not\left(\delta_{2}\right]$,

and

(ii) $\left[\delta_{1}\right) \subseteq\left[\gamma_{1}\right)$ implies $m \not\left[\gamma_{2}\right] ;\left(\delta_{2}\right] \subseteq\left(\gamma_{2}\right]$ implies $m \notin\left[\gamma_{1}\right]$.

Proof: Let the conditions (i) and (ii) be satisfied and suppose $B, \beta^{\prime}$ are any two blocks of $\pi$. We must show that

$$
S(\beta) \neq \phi, S\left(\beta^{\prime}\right) \neq \phi \text { implies } S(\beta)=S(\beta) .
$$

For any block $\beta$ of $\pi$, let $\hat{\beta}$ denote $\beta$ with $m$ removed. Note that $\hat{\beta}$ is then a block of $\hat{\pi}$ (with respect to the "identity", $\hat{e}$ ).

$$
\text { If } \mathrm{m} \not \subset \beta, \mathrm{S}(\beta) \neq \phi \text {, then } \mathrm{S}(\beta)=\mathrm{S}(\hat{\beta}) \neq \phi \text { so } \mathrm{S}(\hat{\beta})=\mathrm{S}(\hat{\pi})=
$$
$S(\hat{\pi})$, since $\hat{\pi}$ is a neighbor of $\hat{e}$. If $m \in \beta, S(\beta) \neq \phi$, then since $\hat{\pi}$ is a neighbor of $\hat{e}$, either $S(\hat{\beta})=\phi$, or $S(\hat{\beta})=S(\hat{\pi})=S(\hat{\pi})$. In the former case $\hat{B}$ is a common interval of $\hat{e}$ and $\hat{\pi}$ and since $\beta$ is a block with $S(\beta) \neq \phi$, either $m_{1} \varepsilon \hat{\beta}$ or $m_{2} \varepsilon \hat{\beta}$. It follows that either $\beta$ has a subinterval of form $\left(m, \delta_{j}\right)$ where $\delta_{j}$ is a common interval of $\hat{\pi}$ and $\hat{e}$ ending with $m_{1}$, or $\beta$ has a subinterval of form $\left(\delta_{2}^{\prime}, m\right)$, where $\delta_{2}^{\prime}$ is a common interval of $\hat{\pi}$ and $\hat{e}$ beginning with $\mathrm{m}_{2}$. Both situations violate condition (i). Hence

$$
S(\beta) \neq \phi \text { implies } S(\hat{\beta})=S(\hat{\pi}) \text { and } \hat{\pi} \subseteq \hat{\beta} \text {. }
$$


Suppose both $m_{1}, m_{2}$ are defined, i.e. $m \neq 1, n$.

If $\mathrm{m}_{1}, \mathrm{~m}_{2} \varepsilon \hat{\pi}$, then for any block $\beta$ of $\pi, s(\beta) \neq \phi$ implies $\hat{\hat{\pi}} \subseteq \hat{\beta}$, hence $m_{1}, m_{2} \varepsilon \beta$, so $m \in \beta$. Then the sequence $\mu=c l_{\pi}(\hat{\pi}, m)$ is a block of $\pi, \mu \subseteq \beta$, and $s(\mu)=s(\beta)$, so (3) holds.

If $\mathrm{m}_{1} \varepsilon \hat{\vec{\pi}}, \mathrm{m}_{2} \not \hat{\vec{\pi}}$, then $\mathrm{m}_{1}$ is maximal in $\hat{\pi}$, so $\mathrm{m}_{2} \varepsilon \gamma_{2}$ and in fact $m_{2}$ is the initial "dot" of $\gamma_{2}$, so $\delta_{2}=\gamma_{2}$. Then conditions

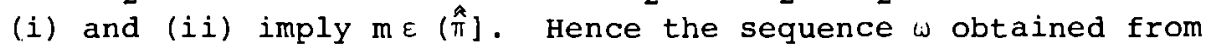
$\hat{\pi}$ by re-inserting $m$ in $(\hat{\pi}]$ is a block of $\pi$ and $m$ is its maximal element. If $m$ is not the last element of the sequence $\omega, i . e$. if $\mathrm{m} \in(\hat{\pi})$, then for any block $\beta$ of $\pi, s(\beta) \neq \phi$ implies $\hat{\pi} \subseteq \hat{\beta}$, so $\omega \subseteq \beta$ and $s(\omega)=s(\beta)$, so (3) holds. If $\mathrm{m}$ is the last element of $\omega$, then maximal in $\omega$ implies $\hat{\pi}$ is also a block of $\pi$ and $s(\omega)=$ $S(\hat{\pi})$. By choice of $\hat{\pi}$ we then have $\hat{\pi} \subseteq \beta$ and $S(\hat{\pi})=S(\beta)$ for any block $\beta$ of $\pi$ such that $S(\beta) \neq \phi$, and again (3) holds. A similar argument applies if $\mathrm{m}_{1} \notin \hat{\pi}, \mathrm{m}_{2} \varepsilon \hat{\pi}$.

Finally, if $\mathrm{m}_{1} \notin \hat{\hat{\pi}}, \mathrm{m}_{2} \not \hat{\pi}$, then by choice of $\hat{\pi}, \mathrm{m}_{1}$ is adjacent to $\mathrm{m}_{2}$ in $\hat{\pi}$ and both are in $\gamma_{1}$ or in $\gamma_{2}$; say without loss of generality $\mathrm{m}_{1}, \mathrm{~m}_{2} \in \gamma_{1}$. Then $\left[\gamma_{1}\right]=\left[\delta_{1}\right) \circ\left(\delta_{2}\right]$, and conditions $(i)$ and (ii) imply that in $\pi$, either $m$ is between $m_{1}$ and $m_{2}$, or $m \varepsilon(\hat{\pi})$. In the former case $\hat{\pi}$ is also a block of $\pi$ and it is clearly the unique nontrivial block. In the latter case, let $\beta$ be any block of $\pi$ such that $S(\beta) \neq \phi$. Then $\hat{\pi} \subseteq \beta$ hence $m \varepsilon \beta$; hence $m_{2} \varepsilon \beta$ (since $\beta$ is a block and the elements of $\hat{\pi}$ are larger than $m$ ). Therefore $\nu=c \ell_{\pi}\left(m_{2}, \hat{\pi}\right)$ is a block of $\pi, \beta \supseteq v$, and $S(\beta)=S(\nu)$, so (3) holds.

In case $m=n, \delta_{2}$ is empty and $m \not\left[\gamma_{1}\right]$ by condition (ii). Moreover we must have $m_{1} \in \hat{\pi}$, since if not then $\gamma_{1}=\hat{\pi}=\hat{e}$ contrary to the assumption that $\hat{\pi}$ is a neighbor of $\hat{e}$ (and hence $\hat{\pi} \neq \hat{e})$. The argument of the second paragraph preceding then implies (3). Similar remarks hold if $\mathrm{m}=1$. The converse is left to the reader.

Given $\hat{\pi}$, we represent slots by the symbol 0 ; we also (where relevant) mark a bracket with an arrow " $\rightarrow$ " if it coincides with the left end of $\hat{\pi}$, and with an arrow " + " if it coincides with the right end of $\hat{\pi}$. The proof of Lemma 3.1 then actually shows that 
the "forbidden" slot-dot sequences for m must have one of the following forms relative to $\hat{\pi}$ :

$$
\begin{aligned}
& \ldots\left[0 \ldots m_{1}\right) \stackrel{\neq \phi}{\ldots} \circ\left(m_{2} \ldots o\right] \ldots \\
& \stackrel{\neq \phi}{\cdots 0}\left(m_{2} \ldots o\right] \ldots\left[0 \ldots m_{1}\right) \circ \stackrel{\neq \phi}{\cdots} \\
& \rightarrow[0 \ldots \phi 0] \cdot \stackrel{\neq \phi}{\gamma_{1} \neq \phi} \cdot\left[0 \ldots m_{1}\right) 0 \stackrel{\neq \phi}{\ldots}, \ldots\left(m_{2} \ldots o\right] \leftarrow
\end{aligned}
$$

or

$$
\rightarrow\left[0 \ldots m_{1}\right) \circ \overbrace{\cdots \infty}^{\neq \phi}\left(m_{2} \ldots \circ\right] \stackrel{\neq \phi}{\neq \cdots}[0 \ldots 0] \leftarrow
$$

The indicated sequences in every case are dot disjoint, and, as dot sequences, are subintervals of $e$. of course, if some $m_{i}^{\prime}$ does not exist, the corresponding half open sequence is empty. Since in any case some slot is not forbidden, we have the following result.

(5) Corollary. For any $m, 1 \leq m \leq n$, let $\hat{e}$ be as above and let $n$ be a permutation on the $n-1$ symbols $\{1, \ldots, n\}-\{m\}$ that is a neighbor of $\hat{\hat{\theta}}$. Then there is some slot in $n$ in which $\mathrm{m}$ can be inserted such that the resulting permutation on $n$ symbols is a neighbor of $e$.

\section{THE DIAMETER OF $\mathrm{P}_{\mathrm{n}}$}

Theorem 3. For $\mathrm{n} \geqq 2$ the diameter of $\mathrm{P}_{\mathrm{n}}$ is two.

Proof. The proof is constructive, and proceeds by induction on $\mathrm{n}$. For $\mathrm{n}=2,3$ it is obvious.

(6) Suppose then that the result is proved for $2 \leq n^{\prime}<n$, $\mathrm{n} \geqq 4$, and let $\sigma$ be a permutation on $n$ symbols such that $\sigma \neq e$ and $\sigma$ is not a neighbor of e.

We say that the permutation $\sigma$ is degenerate if

(i) $\sigma$ and e have a common interval of length $\geqq 2$, 
or

(ii) $\sigma$ and e have the same first symbol or the same last symbol.

If $\sigma$ is degenerate as in case (i) then by treating $\gamma$ as a new symbol we obtain permutations $\widetilde{\sigma}$ and ẽ on $n-|\gamma|+1$ symbols such that $\tilde{\sigma} \neq \tilde{e}$ and $\tilde{\sigma}$ is not a neighbor of $\tilde{e}$. Hence by induction $\tilde{\sigma} \sim \tilde{\pi} \sim \tilde{e}$ for some $\tilde{\pi}$ and it follows that $\sigma \sim \pi \sim$ e for the corresponding $\pi$ on $\{1,2, \ldots, n\}$. Induction also yields the theorem if $\sigma$ is degenerate as in case (ii).

Hence we may assume in the sequel that (6) holds and $\sigma$ is nondegenerate.

(7) We say that a symbol $\mathrm{m}$ is inadmissible in $\sigma$ if

(i) $\mathrm{m}$ is the predecessor* in $\sigma$ of $\mathrm{m}-1$ or the successor in $\sigma$ of $\mathrm{m}+1$;

or

(ii) $\mathrm{m}=1$ and 1 is last in $\sigma$ or $\mathrm{m}=\mathrm{n}$ and $\mathrm{n}$ is first in $\sigma$; or

(iii) $m$ is the predecessor in $\sigma$ of some block $\beta^{\prime}$, where $\beta^{\prime}$ is a rearrangement of $e^{\prime}=(m-j, \ldots, m-1)$ for some $j \geqq 3$ and $\beta^{\prime}$ is a neighbor of $e^{\prime}$;

or

(iv) $m$ is the successor in $\sigma$ of some block $\beta^{\prime \prime}$, where $\beta "$ is a rearrangement of $\mathrm{e}^{\prime \prime}=(\mathrm{m}+1, \ldots, \mathrm{m}+\mathrm{k})$ for some $\mathrm{k} \geqq 3$ and $\beta^{\prime \prime}$ is a neighbor of $e^{\prime \prime}$.

For any given symbol $\mathrm{m}, 1 \leqq \mathrm{~m} \leqq \mathrm{n}$, let $\hat{\pi}, \hat{e}$, etc. be defined as in section 6 .

Lemma 3.2. Suppose $\mathrm{n} \geqq 4, \sigma$ is nondegenerate, and $\mathrm{m}$ is admissible. If $\hat{\sigma} \sim \eta \sim \hat{e}$ for some permutation $\eta$ on symbols $\{1,2, \ldots, n\}-\{m\}$, then there is a slot of $n$ into which $m$ can be inserted, such that the resulting $\eta^{\prime}$ satisfies $\sigma \sim \eta^{\prime} \sim$ e.

The proof is given later in this Report.

$\overline{*_{i . e .}, \text { the immediate }}$ predecessor. 
Any permutation $\sigma$ that can be partitioned into subintervals $\sigma=\delta_{1} \delta_{2} \ldots \delta_{\mathrm{r}}$ such that

$$
\begin{aligned}
& \delta_{1}=\phi \text { or } \delta_{1}=(n), \\
& \delta_{r}=\phi \text { or } \delta_{r}=(1),
\end{aligned}
$$

and, for $1<\mathrm{p}<\mathrm{r}$,

$$
\delta_{p} \text { is an interval of } \bar{e}=(n, n-1, \ldots, 2,1),\left|\delta_{p}\right| \geq 2 \text {, }
$$

will be called a quasi-inverse of $\mathrm{e}^{*}$. ( $\overline{\mathrm{e}}$ is the inverse of $\left.\mathrm{e}.\right)$

Lemma 3.3. If $\mathrm{n} \geqq 4$, $\sigma$ is nondegenerate, and every $m$ is inadmissible in $\sigma$, then $\sigma$ is a quasi-inverse of e. (The proof is given below.)

Theorem 3 now follows from the following explicit construction.

Lemma 3.4. Let $\sigma$ be a quasi-inverse of $e$, where $e=(1,2, \ldots, n)$ and $n \geqq 4$ :

(i) if $\mathrm{n}$ is even, and 1 is not the predecessor of $n$ in $\sigma$, then $\pi=(2,4, \ldots, n, 1,3, \ldots, n-1)$ is a neighbor of both $\sigma$ and $e$;

(ii) if $\mathrm{n}$ is even, and 1 is the predecessor of $\mathrm{n}$ in $\sigma$, then $\pi=(1,3, \ldots, n-1,2,4, \ldots, n)$ is a neighbor of both $\sigma$ and $e$;

(iii) if $\mathrm{n}$ is odd, and 1 is the predecessor of $\mathrm{n}-1$ in $\sigma$, then $\pi=(1,3, \ldots, n, 2,4, \ldots, n-1)$ is a neighbor of both $\sigma$ and $e$;

(iv) if $\mathrm{n}$ is odd, 1 is not the predecessor of $\mathrm{n}-1$ in $\sigma$, and $n$ is not first in $\sigma$, then $\pi=(2,4, \ldots, n-1$, $1,3, \ldots, n)$ is a neighbor of both $\sigma$ and $e$;

*For example, $(8,7,6,3,2,5,4,1)$ is a quasi-inverse of $(1,2,3,4$, $5,6,7,8)$ and it can be partitioned as in (8) by $(8)(76)(32)(54)(1)$ as well as by ()(876)(32)(54)(1). 
(v) if $\mathrm{n}$ is odd, 1 is not the predecessor of $\mathrm{n}-1$ in $\sigma$, and $n$ is first in $\sigma$, then $\pi=(2,4, \ldots, n-1$, $1, n, 3, \ldots, n-2)$ is a neighbor of both $\sigma$ and $e$.

Proof of Lemma 3.4. We illustrate the method of proof for case (i); the others are similar.

Let $\beta$ be any interval of $e,|\beta| \geq 2$, that can be properly rearranged to be an interval $\bar{\beta}$ of $\pi$. By properly we mean $\bar{\beta} \neq$ $\beta$. Then $\bar{\beta}$ contains an odd number and an even number, so $1, \mathrm{n} \in \bar{B}$, hence $1, \mathrm{n} \in \beta$ and $\beta=\mathrm{e}$. Thus, $\pi$ and $\mathrm{e}$ are neighbors.

Let $\beta$ be any interval of $\sigma,|\beta| \geq 2$, that can be properly rearranged to be an interval $\bar{\beta}$ of $\pi$. Suppose $|\beta|=2$, say $\beta=$ $\left(j, j^{\prime}\right)$. We cannot have $\left|j-j^{\prime}\right|=1$, because then $\bar{\beta}$ would not be an interval of $\pi$. Hence by $(8), j$ is the last element of some $\delta_{p^{\prime}} j^{\prime}$ the first in $\delta_{p+j}$. Since $\bar{\beta}$ is an interval of $\pi$, either $\left|j-j^{\prime}\right|=2$ or $\left(j, j^{\prime}\right)=(n, 1)$. In the former case $\sigma$ could not be a quasi-inverse of $\mathrm{e}$. In the latter case $\mathrm{n}$ precedes 1 in $\sigma$ (by case (i) hypothesis), so $\bar{\beta}$ is not a proper rearrangement of $\beta$.

We may therefore assume that $|\beta| \geqq 3$. Since $\sigma$ is a quasiinverse, $n \geq 4, \beta$ contains some interval of form $(k, k-1)$. But then $c l_{\pi}(k, k-1) \subseteq \beta$, and by construction of $\pi$,

(9) for any consecutive integers $j, j^{\prime}$ in e at least one of $j, j^{\prime} \varepsilon \beta$.

Moreover, $1, \mathrm{n} \varepsilon \beta$. Suppose $2 \not \beta$. If 2 succeeds $\beta$ then 1 must also succeed 2 , whence $1 \not \beta$, contradicting (9), hence 2 precedes $\beta$ and (since $n \varepsilon \beta$ ) we must have $\sigma=(2, \Gamma \beta \ldots)$. In particular, $4 \varepsilon \beta$, for otherwise there is no position for 3 consistent with $\sigma$ being a quasi-inverse. Similarly, if $n-1 \not B$, then $n-1$ is last in $\sigma$, and $n-3 \varepsilon \beta$. Therefore $\sigma=(2, \beta, n-1)$ or $\sigma=(2, \beta)$ or $\sigma=(\beta, n-1)$, or $\sigma=\beta$ so $\pi$ is a neighbor of $\sigma . \square$

Proof of Lemma 3.3. Let (i), (ii), (iii) or (iv) hold in (7) for every $\mathrm{m}, 1 \leq \mathrm{m} \leq \mathrm{n}$. Suppose that neither (i) nor (ii) holds for some particular $m$. Say $\beta "$ is as in (iv) for $m$ (a similar argument holds if m satisfies (iii) with $\beta^{\prime}$ ). 
Let $m+j$ be the predecessor of $m$ in $\sigma, m+j \neq m+1$ by hypothesis. Since $m+j$ is inadmissible, is neither first nor last in $\sigma$, and cannot satisfy (iii) we have that either

(a) $m+j$ is inadmissible by (iv),

or

(b) $m+j$ is the successor of $m+j+1$.

In case (a) $m+j$ must be the successor of a block $\gamma$ which is a rearrangement of $(m+j+1, \ldots, m+j+l), \ell \geqq 3$. clearly $\gamma \subseteq \beta$ ", since $m+1 \not \gamma$ but precedes $m+j$ in $\beta^{\prime \prime}$. Since $\beta^{\prime \prime}$ is a neighbor of $e^{\prime \prime}$, and $(\gamma, m+j)$ is a nontrivial subblock of $\beta ", \gamma$ must be an interval of $e$, that is $\gamma=(m+j+1, \ldots, m+j+l)$. But then $\sigma$ is degenerate, a contradiction.

Therefore (b) holds. Again, since $\beta^{\prime \prime}$ is a neighbor of $e^{\prime \prime}$, and $(m+j+1, m+j)$ is a nontrivial subblock of $\beta "$, we conclude that the predecessors of $m+j+1$ in $b^{\prime \prime}$ form an interval $\beta^{\prime \prime}$ of $e$. Then $\sigma$ is degenerate unless $\left|\beta^{\prime \prime}\right|=1$. Thus $\beta^{\prime \prime}=(\mathrm{m}+1, \mathrm{~m}+3, \mathrm{~m}+2, \mathrm{~m})$. But then $m+1$ is admissible, a contradiction.

Thus 7 (i) or 7 (ii) holds for every $m, 1 \leqq m \leqq n$, from which it follows that $\sigma$ has a decomposition as in (8), i.e. $\sigma$ is a quasi-inverse of $e$. This completes the proof of Lemma 3.3 .

Proof of Lemma 3.2. Let $\mathrm{n} \geqq 4, \sigma$ nondegenerate, m admissible, and suppose $\hat{\sigma} \sim \eta \sim \hat{e}$. We cannot have $\hat{\sigma}=\hat{e}$ (since $\sigma$ is nondegenerate). If $\eta=\hat{e}$ or $\eta=\hat{\sigma}$, then the corollary to Lemma 3.1 implies there is an $\eta^{\prime}$ such that $\sigma \sim \eta^{\prime} \sim$ e. Hence we may assume that

$$
\hat{\sigma}, \eta, \hat{e} \text { are distinct. }
$$

By Lemma 3.1 , the collection $\zeta_{e}$ of forbidden slot-dot sequences in $n$ are of form (A) or (B). That is, if $m$ is put in a slot which is in no sequence $s \in \mathscr{C}_{e}$ then the resulting $\eta^{\prime}$ is a neighbor of e. Symmetrically, there is a collection $C_{\sigma}$ of slotdot sequences in $n$ having form (A) or (B) (with $m_{i}$ replaced by $m_{i}^{\prime}, i=1,2$ ) such that if $m$ is put in a slot which is in no 
$s \in \mathscr{C}_{\sigma}$ then $\eta^{\prime}$ is a neighbor of $\sigma$. To show that there is some $\eta^{\prime}$ that is a neighbor of both e and $\sigma$, it suffices to show that



Suppose both $\mathscr{C}_{e}$ and $\mathscr{C}_{\sigma}$ are of type (A). The sequences (there are at most two of them) in $\mathcal{E}_{e}$ are dot-disjoint, as are the sequences in $\mathscr{C}_{\sigma}$. Moreover, each has equally many slots and dots. But $n$ has $n$ slots and $n-1$ dots, hence if (11) is false, there is some $s \in \mathcal{E}_{e^{\prime}} \underline{\sim}^{\prime} \varepsilon \mathcal{G}_{\sigma}$ such that $\underset{\sim}{s} \sim_{\sim}^{\prime}$ contains more dots than slots. But if $\mathbf{s}_{\sim} \cap \mathbf{s}_{\sim}^{\prime}$ contains $\geq 2$ dots, then the dots in $\underset{\sim}{s} \cap s_{\sim}^{\prime}$ constitute a common interval of e and $\sigma$, contrary to the nondegeneracy of $\sigma$. Hence $\mathbf{s}_{\sim} \cap_{\sim}^{\prime}$ consists of one dot, but then this dot must be $m_{i}=m_{j}^{\prime}$ for some $i$ and $j$, contradicting the hypotheses that $\sigma$ is nondegenerate and $\mathrm{m}$ is admissible.

Therefore we may assume that at least one of $\mathscr{C}_{e}, \mathscr{C}_{\sigma}$ is of type B, say without loss of generality that $\mathscr{C}_{\sigma}$ has form B1:

$$
\rightarrow[0 \ldots 0] \underbrace{\overbrace{\ldots}^{u} \cdot\left[0 \ldots m_{1}^{\prime}\right) \sigma^{x}, \ldots o}_{\beta}\left(m_{2}^{\prime} \ldots o\right]+, \quad u \neq \phi, v \neq \phi .
$$

(13) Suppose, by way of contradiction, that every slot is forbidden by sequences in either $\mathscr{C}_{\sigma}$ or $\mathscr{C}_{\mathrm{e}}$. Suppose also that $\left[\delta_{1}\right)=\left[0 \ldots m_{1}\right) \in \mathscr{B}_{e}$ meets the slot-dot sequence $\mathrm{v}$. If $\left[\delta_{1}\right) \cap$ $\left(m_{2}^{\prime} \ldots 0\right] \neq \phi$, then either $m_{1}=m_{2}^{\prime}$, or they overlap in $\geq 2$ dots; the first is impossible because $\mathrm{m}$ is admissible, the second violates the assumption that $\sigma$ is nondegenerate. Therefore the slot immediately to the right of $\left[\delta_{1}\right)$ is in $v$ and not forebidden by $\mathscr{C}_{\mathrm{e}}$, a contradiction.

If $\mathscr{B}_{e}$ is of type (A), this means that $v \subseteq\left(\delta_{2}\right]=\left(m_{2} \ldots o\right]$. Since $\left.m_{2} \not 0 \ldots m_{1}^{\prime}\right)$, it then follows that the slot immediately to the left of $\left(\delta_{2}\right]$ is not forbidden by $\mathscr{C}_{e}$ or $\mathscr{C}_{\sigma}$ (a contradiction) unzess $B \subseteq\left(\delta_{2}\right]$. Now $\hat{\sigma}$ is obtained from $n$ by rearranging only the elements within $\beta$. Let $e "$ be the subinterval of $\delta_{2}$ beginning 
with $m_{2}=m+1$ and ending with the last element of $\beta$. $e "$ is by definition a subinterval of $e$, and its rearrangement, $\beta "$, in $\hat{\sigma}$ is a neighbor of $e^{\prime,}$ because $\hat{\sigma}$ is a neighbor of $\eta$. Moreover, $m_{1}^{\prime}$ is the last element in the rearrangement $B^{\prime \prime}$, and by definition $m$ is the successor in $\sigma$ of $\beta^{\prime \prime}$. If $\left|\beta^{\prime \prime}\right|=2$ then $\beta^{\prime \prime}=e^{\prime \prime}=\left(m_{2}, m_{j}^{\prime}\right)$ and $\eta=\hat{\sigma}$, contrary to assumption. Hence $\left|\beta^{\prime \prime}\right| \geqq 3$ and $\left(\beta^{\prime \prime}, m\right)$ is a subsequence of o satisfying (iv), whence $m$ is inadmissible, a contradiction.

If $\mathscr{C}_{\mathrm{e}}$ is of type (B1), we have by paragraph (13) that $v \subseteq\left[\gamma_{1}\right] \cup\left(\delta_{2}\right]$. If $v \subseteq\left(\delta_{2}\right]$, then, as above, we find that $\beta \subseteq\left(\delta_{2}\right]$, which results in the same sort of contradiction. The case $v \subseteq\left[\gamma_{1}\right]$ is seen to be symmetric by interchanging the roles of $\sigma$ and $e$.

Suppose $\mathscr{C}_{e}$ is of type (B2). If $m_{2}^{\prime}$ exists and $m_{2}^{\prime} \varepsilon\left[\gamma_{2}\right]$, then $\mathrm{n}$ is last in both $\sigma$ and $e$, contradicting nondegeneracy. Therefore if $m_{2}^{\prime}$ exists, $v \subseteq\left(m_{2} \ldots o\right)$ by paragraph (13), hence $\beta \subseteq\left(m_{2} \ldots o\right)$ and a contradiction is obtained as before. We may therefore assume that $m_{2}^{\prime}$ does not exist, and, symmetrically, that $m_{1}$ does not exist. But then $m=1$ was last in $\sigma$, so $m$ was inadmissible, a contradiction. $\square$

\section{REFERENCES}

[1] Bowman, V.J., Permutation Polyhedra, SIAM Jour. Appl. Math., 22 (1972), 580-589.

[2] Even, S., A. Lempel, and A. Pnueli, Permutation Graphs and Transitive Graphs, Jour. ACM, 19 (1972), 400-410.

[3] Even, S., A. Lempel, and A. Pnueli, Transitive Orientation of Graphs and Identification of Permutation Graphs, Can. Jour. Math., 23 (1971), 160-175.

[4] Gilmore, P.C. and A.J. Hoffman, A Characterization of Comparability Graphs and of Interval Graphs, Can. Jour. Math. , 16 (1964), 539-548.

[5] Young, H.P. and A. Levenglick, A Consistent Extension of Condorcet's Election Principle, RR-77-15, International Institute for Applied Systems Analysis, Laxenburg, Austria, 1977; to appear also in SIAM Jour. Appl. Math. - Part $C$.

[6] Johnson, S.M., Generation of Permutations by Adjacent Transposition, Mathematics of Computation, 17. (1963), 282-285.

[7] Trotter, H.F., Algorithm 115, Comm. ACM, $\underline{5}$ (1962), 434-435. 
FELATED IIASA PUBLICATIONS

Sensitivity Analysis of Streeter-Phelps Models. S. Rinaldi, R. Soncini-Sessa. (RR-77-001) $\$ 1.50$ AS 30 .

On Huntington Methods of Apportionment. M.L. Balinski, H.P. Young. (RR-77-OO2) \$1.50 AS30.

An Algorithm for Constrained Optimization with Semismooth Functions. R. Mifflin. (RR-77-003) $\$ 2.50$ AS45.

Reservoirs with Seasonally varying Markovian Inflows and their First Passage Times. E.H. Lloyd. $(R R-77-004) \$ 2.50$ AS45.

Power, Prices, and Incomes in Voting systems. H.P. Young. (RR-77-005) \$1.50 AS30.

Polyhedral Dynamics and the Geometry of Systems. R. Atkin, J. Casti. (RR-77-006) \$2.50 AS45.

Gptimal Allocation of Artificial In-Stream Aeration. S. Rinaldi, R. Soncini-Sessa. (RR-77-007) $\$ 1.50$ AS30.

Dual Systems of Dynamic Linear Programming. A. I. Propoi. (RR-77-0O9) \$1.50 AS30.

uuotatone Apportionment Methods. M.L. Ealinski, H.P. Young. (RR-77-O11) \$1.5C AS3O.

Lobbying and Campaigning with Applications to the Measure of Power. H.P. Young. (RR-77-013) \$2.50 AS45.

A Consistent Extension of Condorcet's Election Frinciple. H.P. Young, A. Levenglick. $(\mathrm{RR}-77-015) \quad \$ 1.50$ AS 30 .

On the Distribution of the Hurst Range of Independent Normal Summands. A.A. Anis, E.H. Lloyd. $(\mathrm{RR}-77-\mathrm{C} 16) \$ 1.50$ AS 30 . 
Stability, Coalitions, and Schisms in Proportional Representation Systems. M.L. Balinski, H.P. Young. (RR-77-017) \$1.50 AS30.

Stochastic Control for Linear Discrete-Time Listributed-Lag Models. W.E. Arthur. (RR-77-018) $\$ 1.00$ AS20.

On Measures of Natural Fesource scarcity. A.C. Fisher. (RR-77-019) \$1.50 AS30.

Software Package for Economic Modelling. M. Norman. (RK-77-021) \$5.40 AS95.

Macrodynamics of Technological Change: Market Penetration by New Technologies. V. Peterka. (RR-77-022) $\$ 5.60$ AS100.

Please cite publication number when making an order. See inside back cover for order information. 